$>$ La plupart des bactéries utilisent un système de communication, le quorum sensing, fondé sur la sécrétion et la perception de petites molécules appelées autoinducteurs qui leur permettent d'adapter leur comportement en fonction de la taille de la population. Les bactéries mutualisent ainsi leurs efforts de survie en synchronisant entre elles la régulation de gènes impliqués notamment dans la virulence, la résistance aux antimicrobiens ou la formation du biofilm. Des méthodes ont vu le jour pour inhiber cette communication entre bactéries et limiter leurs effets nocifs. Des inhibiteurs chimiques, des anticorps ou encore des enzymes capables d'interférer avec les autoinducteurs ont été développés et se sont montrés efficaces pour diminuer la virulence des bactéries à la fois in vitro et in vivo. Cette stratégie, appelée quorum quenching, a également montré des effets synergiques avec des traitements antibactériens classiques. II permettrait notamment d'augmenter la sensibilité des bactéries aux antibiotiques. Ceci constitue une piste thérapeutique prometteuse pour lutter contre les infections bactériennes et limiter les conséquences de l'antibiorésistance. <

Les bactéries sont des organismes unicellulaires ubiquitaires qui entretiennent de nombreuses interactions avec leur environnement. Elles intègrent une multitude de stimulus abiotiques (comme les nutriments, la température) et biotiques (comme les bactériophages, l'hôte, les bactéries compétitrices) via des systèmes variés de détection leur permettant d'adapter leur comportement à leur environnement. Les bactéries étaient considérées comme des entités individuelles et «asociales », jusqu'à la découverte d'un système de communication interbactérien, nommé quorum sensing (QS) [1-3]. Dès 1965, Alexander Tomasz observe que l'induction de la compétence chez Streptococcus

Vignette (Photo @ Inserm/CEA - Philippe Huber et Stéphanie Bouillot).

\section{Quorum sensing et quorum quenching \\ Comment bloquer la communication des bactéries pour inhiber leur virulence?}

\begin{abstract}
Sonia Mion ${ }^{1}$, Benjamin Rémy ${ }^{1,2}$, Laure Plener ${ }^{2}$,
\end{abstract} Éric Chabrière ${ }^{1}$, David Daudé2

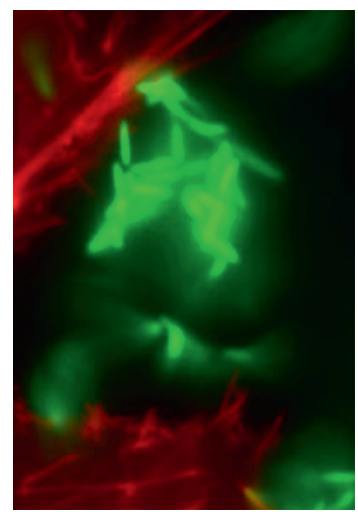

${ }^{1}$ Aix Marseille Univ, Institut de recherche pour le développement (IRD), Assistance PubliqueHôpitaux de Marseille (APHM), Microbes évolution phylogénie et infections (MEPHI), Institut hospitalo-universitaire (IHU)Méditerranée Infection, 13005 Marseille, France.

${ }^{2}$ Gene\&GreenTK, 19-21, boulevard Jean Moulin, 13005 Marseille, France. david.daude@gene-greentk.com

pneumoniae (anciennement Pneumococcus) peut s'effectuer sans contact direct entre bactéries, mais par l'intermédiaire d'une molécule diffusible qu'elles produisent [4]. Plus tard, en 1970, Vibrio fischeri (anciennement Photobacterium fischeri) a fait l'objet d'observations similaires à propos de la régulation de la bioluminescence émise par ces bactéries [5] : la fabrication de photons est en effet induite uniquement à partir de la phase exponentielle de croissance des bactéries et non dans une culture fraîchement inoculée lorsque la densité cellulaire est faible. Les auteurs ont alors émis l'hypothèse d'une autoinduction pour la régulation de ce processus et ils ont nommé autoinducteur (Al) la molécule régulatrice qui s'accumule dans le milieu $[6,7]$. Le terme de quorum a, par la suite, été introduit pour décrire l'idée d'un nombre minimal de bactéries nécessaire au déclenchement de comportements de groupe, comme la production de bioluminescence [8]. Au début du Xxl ${ }^{e}$ siècle, le QS a été identifié chez de nombreuses bactéries à Gram positif et négatif. II intervient dans la régulation de processus variés comme la sporulation, la virulence, la formation de biofilm ou encore la synthèse d'antibiotiques [9].

\section{Mais qu'est-ce que le quorum sensing?}

Le quorum sensing (QS) est un mode de communication et de perception utilisé par les bactéries. II se fonde sur la production de petites molécules, les autoinducteurs (AI), qui peuvent diffuser à travers la membrane ou être transportés à l'extérieur de la cellule (Figure 1) [1]. Les Al, dont la concentration est proportionnelle au nombre de bactéries, servent d'indicateur moléculaire de la densité bactérienne. À par- 


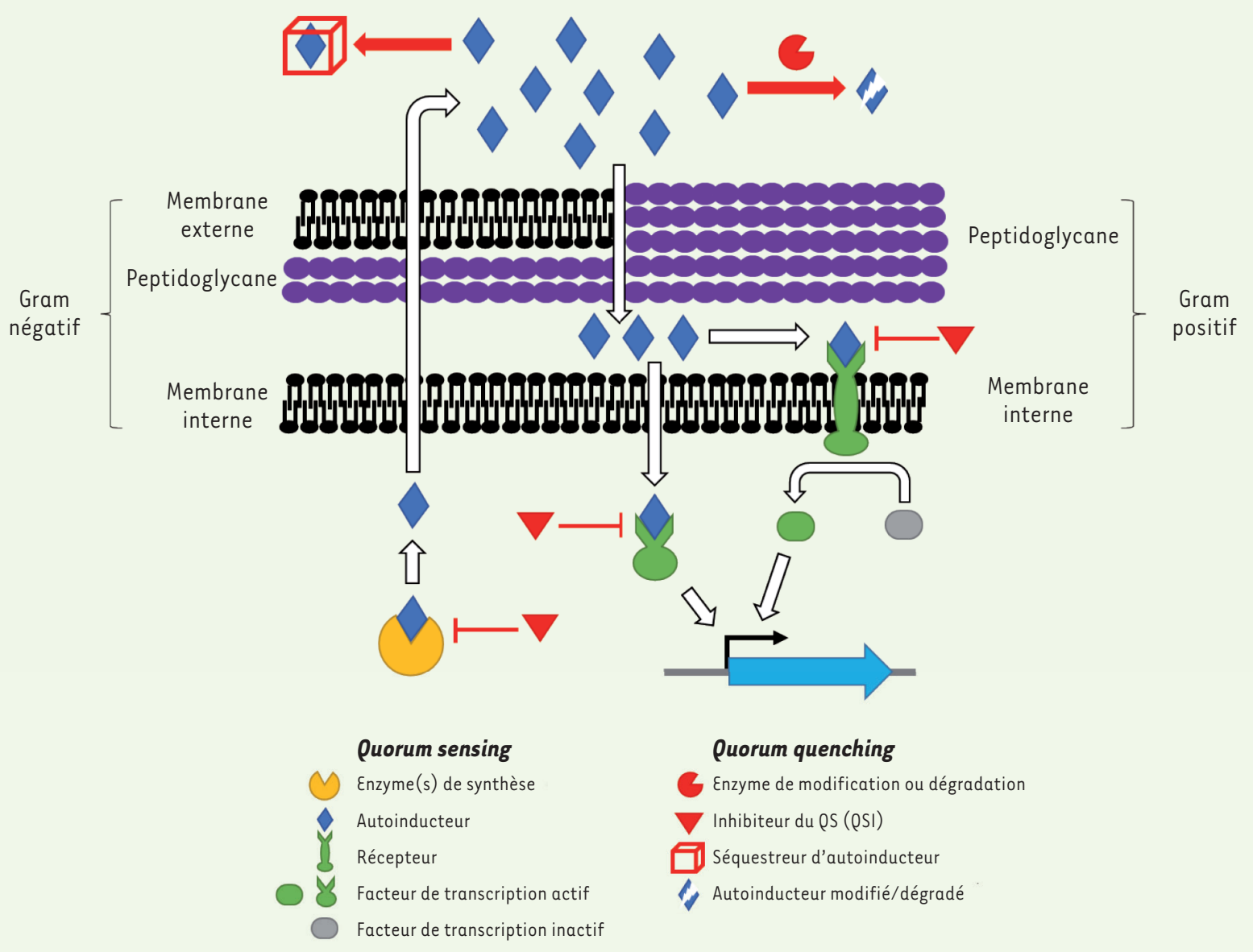

Figure 1. Mécanismes du quorum sensing bactérien et des différentes stratégies de quorum quenching. Le quorum sensing consiste en la production et la perception de molécules signal par les bactéries. La perception de ces molécules peut être extracellulaire, par des senseurs membranaires qui induisent une cascade de phosphorylation, ou intracellulaire, par des régulateurs de transcription. Dans les deux cas, le quorum sensing provoque une activation de certains gènes propices à des comportements de groupe des bactéries. Le quorum quenching consiste en l'interruption du quorum sensing. II peut agir au niveau extracellulaire par la dégradation ou séquestration des molécules signal ou intracellulaire avec des inhibiteurs du quorum sensing (QSI).

tir d'une certaine concentration de ces molécules, une réponse cellulaire est enclenchée par l'activation et la répression de gènes efficaces seulement lorsque la densité cellulaire est importante, pour mettre en place des phénotypes particuliers, comme la formation de biofilm, la virulence, la production d'exopolysaccharides, d'exoprotéases et de sidérophores. De nombreux facteurs induits par le QS sont sécrétés dans l'environnement de la cellule. Ils ont un intérêt global pour la communauté bactérienne pour fournir des nutriments à la population ou pour la transition du mode de vie planctonique (c'est-à-dire en suspension) vers un mode sessile, appelé biofilm, plus fréquemment rencontré en milieu naturel [10] $(\rightarrow)$.

$(\rightarrow)$ Voir la synthèse de A. Filloux et $\mathrm{I}$. Vallet, $\mathrm{m} / \mathrm{s} \mathrm{n}^{\circ} 1$, janvier 2003, page 77

Le QS ne servirait pas uniquement au recensement de la population bactérienne, mais également à la perception de la diffusion des facteurs sécrétés dans le milieu, pour optimiser l'efficacité de leur production [11]. Pour intégrer au mieux ces différentes informations, les bactéries ont développé des systèmes de QS impliquant plusieurs
Al, de stabilités et de solubilités différentes, perçus par des récepteurs spécifiques qui sont interconnectés [12]. Par exemple, la bactérie pathogène opportuniste Pseudomonas aeruginosa produit et perçoit quatre Al distincts, chacun étant perçu par un régulateur spécifique [13]. Ces quatre régulateurs sont organisés de manière hiérarchique, avec un régulateur dominant les trois autres. Chez la bactérie marine modèle du QS, Vibrio harveyi, trois Al sont utilisés et les trois signaux qu'ils produisent sont intégrés dans une même voie du QS induisant un gradient dans l'activation des gènes du QS au niveau de la population bactérienne [14]. Ce fonctionnement permet aux bactéries d'avoir un mode de communication combinatoire pour mettre en place une réponse précise aux signaux qu'elles perçoivent et ainsi adapter le mode de vie le plus propice à leur survie [12]. 
AHL

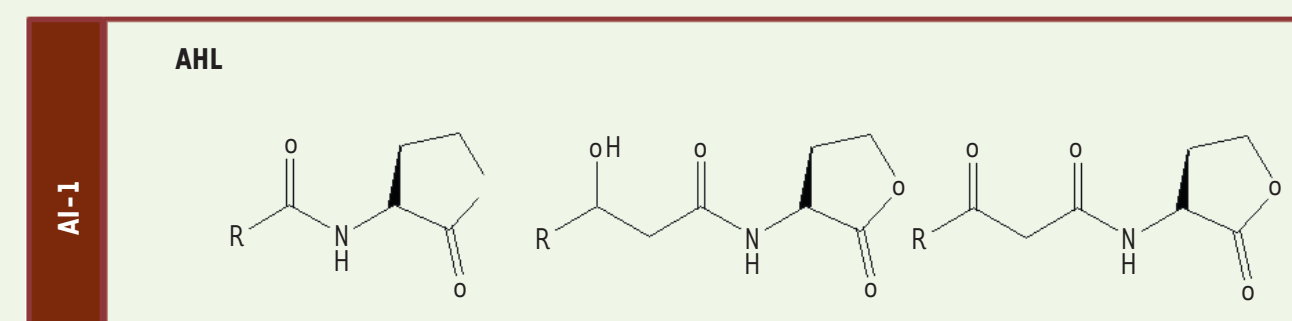

Acinetobacter, Aeromonas, Agrobacterium, Burkholderia, Erwinia, Pseudomonas, Rhizobium, Serratia, Vibrio, Yersinia
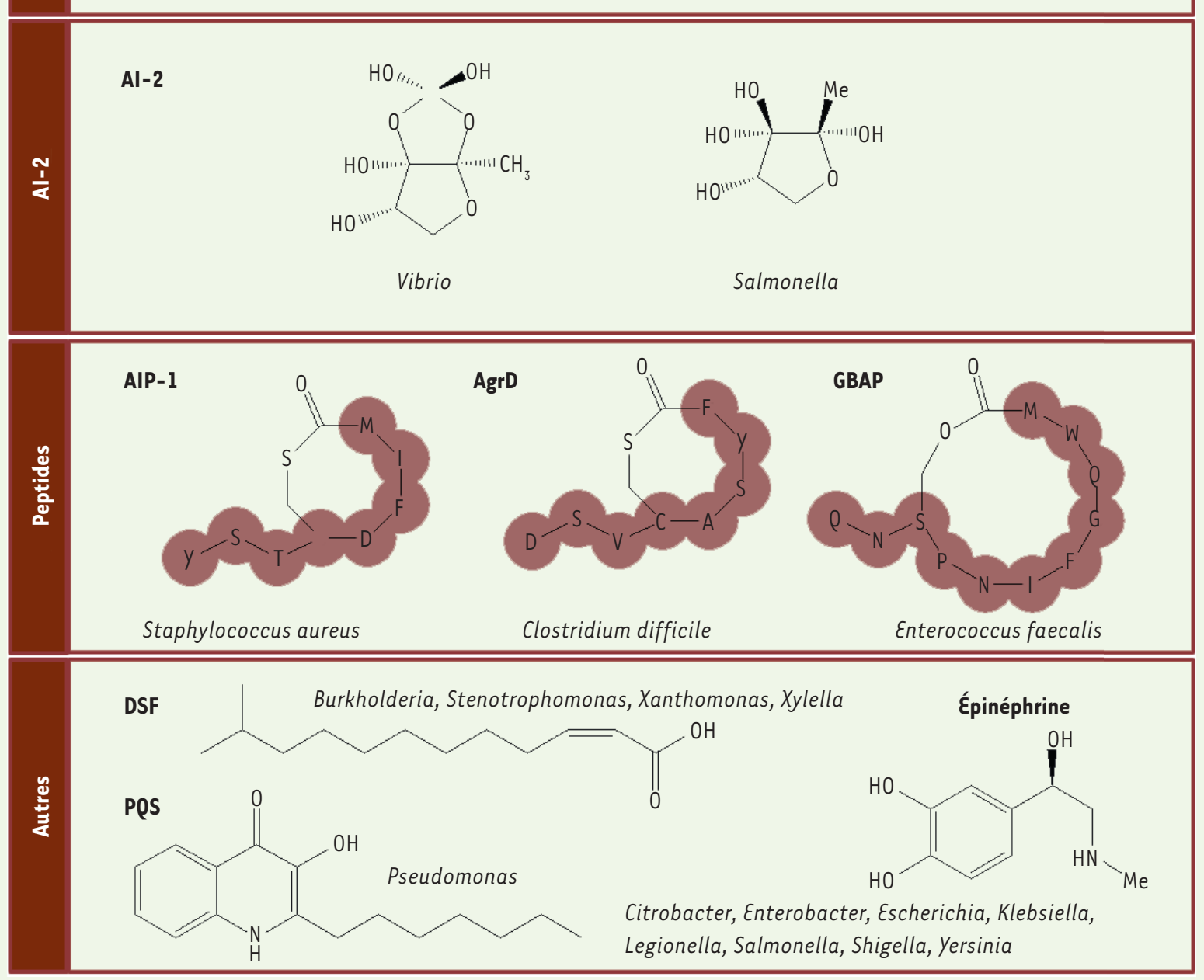

Figure 2. Principales molécules du quorum sensing bactérien. Les bactéries utilisent des molécules de communications variées. La plupart des bactéries à Gram négatif utilisent des acyl homosérines lactones ( $\mathrm{AHL}$ ou autoinducteurs de type I, Al-I). Les autoinducteurs de type 2 (Al-2) sont quant à eux utilisés à la fois par des bactéries à Gram négatif et à Gram positif. Chez ces dernières, on retrouve également des peptides autoinducteurs comme AIP-1 (autoinducing peptide 1) chez Staphylococcus aureus, AgrD (Agr autoinducing peptide) chez Clostridum difficile ou GBAP (gelatinase biosynthesis-activating pheromone) chez Enterococcus faecalis. Enfin, d'autres molécules peuvent être utilisées par les bactéries comme le PQS (Pseudomonas quinolone signal) et le DSF (diffusible signal factor).

\section{Les molécules du quorum sensing}

Il existe une grande diversité de molécules de communication entre bactéries (Figure 2). Les bactéries à Gram positif (Staphylococcus spp., Clostridium spp., Enterococcus spp.) utilisent souvent des pep- tides cycliques d'environ 7 à 11 acides aminés. Ces peptides sont transportés hors de la cellule bactérienne puis sont perçus par le senseur membranaire des autres bactéries (senseur à histidine kinase). La fixation du peptide au senseur entraîne l'activation d'un régulateur 
de réponse (par exemple sa phosphorylation) activant l'expression des gènes du QS [15]. Chez les bactéries à Gram négatif, différentes molécules sont utilisées, les plus communes étant les acyl homosérine lactones ( $\mathrm{AHL}$ ), dont les tailles (de 4 à 18 atomes de carbone) et les modifications de la chaîne acyl varient selon les bactéries. Par exemple, $P$. aeruginosa produit et perçoit, entre autres $A I$, deux $A H L$ : la $\mathrm{N}$-(3-oxododécanoyl)-L-homosérine lactone (3-oxo-C12-HSL) et la $\mathrm{N}$-butyryl-L-homosérine lactone (C4-HSL). Les AHL diffusent librement à travers la membrane bactérienne et la réponse au QS est déclenchée par leur fixation dans la cellule à un régulateur de transcription de type LuxR qui, ainsi activé, induit l'expression des gènes du QS. Parmi les gènes induits, se trouvent le gène de la synthase correspondant à l'Al et le gène codant le récepteur qui lui est associé, permettant ainsi I'autoinduction du QS [14]. D'autres molécules sont impliquées dans le QS des bactéries à Gram négatif. Celles-ci sont spécifiques de chaque espèce bactérienne: des acides gras insaturés, comme le DSF (diffusible signaling factor), sont utilisés par les bactéries Xanthomonas spp., Burkholderia spp. et Xylella spp. ; des cétones (CAI-1 [cholerae autoinducer-1] et LAI-1 [legionella autoinducer-1]) par Vibrio spp. et Legionella spp. ; l'épinéphrine et ses dérivés sont utilisés par les bactéries entérohémorragiques [16]. Le système de QS de $P$. aeruginosa utilise également une quinolone (PQS, pour pseudomonas quinolone signal) qui, à cause de son insolubilité en milieu aqueux, transite entre les cellules par des vésicules membranaires [17]. Un autre Al est utilisé par les bactéries à Gram positif et négatif, le diester Al-2. II existe sous forme borée (chez Vibrio spp.) ou non borée (chez Escherichia coli ou Salmonella spp.). Utilisé par un large spectre de bactéries, il est considéré comme une molécule signal inter-espèces [18].

\section{Comment interférer avec le quorum sensing? Le quorum quenching}

Le quorum sensing ( $Q S$ ) intervient dans l'activation et la régulation de nombreux phénotypes bactériens pouvant être problématiques pour l'homme. Différentes stratégies ont donc été développées pour inhiber ces comportements régulés par le $Q S$ en bloquant la communication entre les bactéries (Figure 1). Cette méthode d'inhibition du QS est appelée quorum quenching $(\rho Q)$. La composante principale du $Q S$ étant la production et la détection de molécules signal, le $Q \rho$ peut interférer de différentes manières avec ce système, soit au niveau intracellulaire, soit au niveau extracellulaire. L'utilisation de molécules inhibitrices, nommées inhibiteurs du QS (PSI), mimant les Al, permet ainsi d'intervenir au niveau des cellules bactériennes pour inhiber la production ou la perception des Al [19]. D'autres stratégies ciblent directement les molécules de communication sécrétées dans l'environnement avant qu'elles n'atteignent leur cible, soit en les séquestrant à l'aide d'anticorps [20] ou de macromolécules comme les cyclodextrines [21], soit en les dégradant avec des enzymes spécifiques (ou $Q \rho \mathcal{E}$ pour quorum quenching enzyme) [22]. Le QQ présente l'avantage de ne pas affecter la croissance des bactéries, contrairement aux antibiotiques, ce qui minimise nettement la pression de sélection et devrait limiter l'apparition de résistance [23]. Le $Q \rho$ représente donc une approche idéale pour la prévention et le traitement des infections bactériennes et pourrait constituer une alternative élégante aux antibiotiques.

\section{Les inhibiteurs du quorum sensing}

De nombreux inhibiteurs du QS (QSI) d'origines variées ont été étudiés. Beaucoup de QSI ont été isolés à partir de produits naturels, comme le thé, les champignons, le miel, l'ail, le clou de girofle, et d'organismes marins [19]. D'autres ont été synthétisés en laboratoire, en mimant la structure des Al pour créer des molécules similaires, mais inactives (antagonistes) [24]. Certains Al comme l'azithromycine étaient déjà utilisés pour leurs propriétés antibiotiques avant que leur potentiel de $\mathrm{Q}$ ne soit découvert. L'azithromycine a été évaluée chez des patients atteints de pneumonie et aurait de potentiels effets bénéfiques pour traiter des infections à $P$. aeruginosa. Le 5 -fluorouracile, une molécule déjà disponible dans le traitement de cancers, a été testé pour ses propriétés $Q Q$. L'efficacité de cette molécule a été évaluée in vitro sur plusieurs phénotypes bactériens régulés par le $Q S$, dont la formation de biofilm chez P. aeruginosa [25]. Hormis quelques QSI, très peu de molécules à visée $\mathrm{Q} \rho$ ont atteint les premières phases d'un essai clinique. Pourtant de nombreuses molécules de $Q Q$ ont montré des effets bénéfiques in vivo dans des modèles animaux comme les amibes, le ver Caenorhabditis elegans et les modèles murins [26].

\section{Les séquestreurs d'Al}

L'utilisation d'anticorps capables de séquestrer les molécules signal a également été considérée. L'anticorps synthétique AP4-24H1l est, par exemple, capable de se lier aux peptides autoinducteurs AIP-4 (autoinducing peptide 4) utilisé par Staphylococcus aureus pour réguler sa virulence et inhiber certains mécanismes, comme son activité hémolytique [20]. D’autres molécules peuvent également être utilisées pour piéger les Al et perturber la communication bactérienne. C'est le cas des cyclodextrines, des oligosaccharides cycliques composées de plusieurs unités D-glucopyranosyle formant une cage moléculaire capable de piéger les AHL et contrôler le QS [21].

\section{Les enzymes à activités $\mathbf{Q P}$}

Les enzymes capables de dégrader les Al (les $Q \rho \varepsilon$, quorum quenching enzyme) ont été découvertes chez la bactérie Bacillus spp. [27]. Depuis, de nombreuses enzymes ont été identifiées, caractérisées et améliorées dans le but de les utiliser comme agents de $\rho \rho$ [22]. Ces enzymes présentent l'intérêt d'agir au niveau extracellulaire et en quantité catalytique (Figure 3). Plusieurs classes de QQE ont été décrites, la plupart ciblant les AHL, comme 


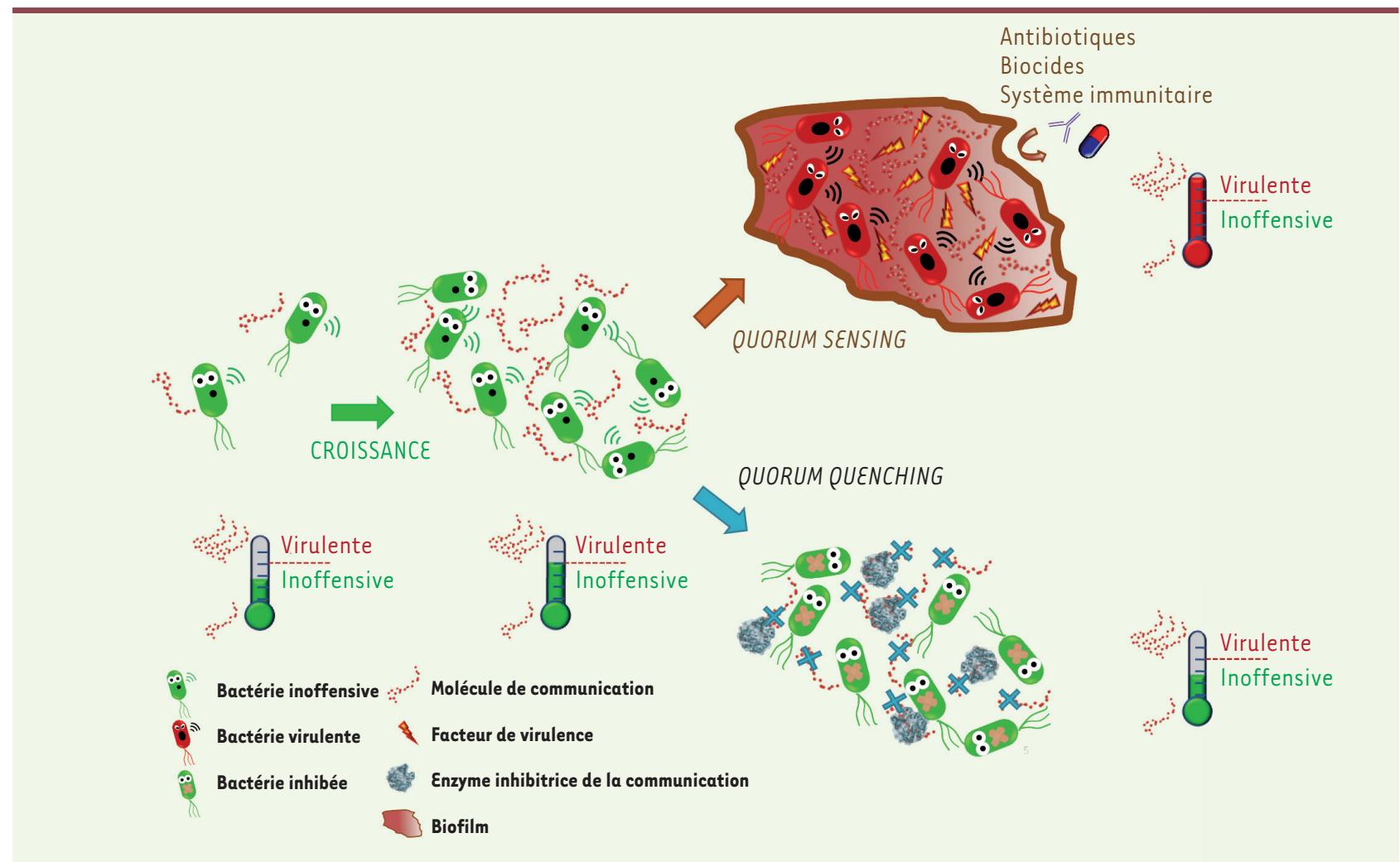

Figure 3. Rôle du quorum sensing dans la mise en place de l'infection et de la protection grâce à l'utilisation d'enzyme inhibitrice. Les bactéries sécrètent dans leur environnement des molécules de communication qui leur permettent de se signaler les unes aux autres. À partir d'une certaine taille de la population, la concentration de molécules de communication va atteindre un seuil au-delà duquel les bactéries vont adopter un comportement de groupe. Ce système de communication, appelé quorum sensing, va permettre aux bactéries de synchroniser leur virulence et la formation du biofilm qui leur permet de se prémunir du système immunitaire de l'hôte et de l'action des agents antibactériens comme les antibiotiques. Pour pallier ce phénomène, une stratégie appelée quorum quenching consiste à brouiller la communication des bactéries pour les empêcher de se synchroniser. Des enzymes peuvent, par exemple, être utilisées pour dégrader les molécules de communication et maintenir les bactéries dans un état inoffensif.

les lactonases, acylases et oxydoréductases [22]. Quelques enzymes agissant sur l'Al-2 et le PQS de P. aeruginosa ont aussi été rapportées, mais elles restent minoritaires [22,28]. La principale limitation de l'utilisation thérapeutique de ces enzymes est liée à leur faible stabilité. Des enzymes issues d'organismes extrêmophiles, capables de résister à de nombreuses contraintes (température, solvants, détergents), ont donc été étudiées [29]. Une attention particulière a été portée à la lactonase hyperthermophile SsoPox (Sulfolobus solfataricus paraoxonase) isolée de l'archée Sulfolobus solfataricus provenant des sources chaudes du Vésuve, pour le $Q \rho$ de $P$. aeruginosa, à la fois in vitro, sur des isolats cliniques, et in vivo, dans un modèle de pneumonie développé chez le rat $[30,31]$. L'administration intratrachéale de l'enzyme, capable de dégrader les AHL, a ainsi permis d'augmenter le taux de survie chez l'animal d'un facteur 4.

\section{Le potentiel thérapeutique du quorum quenching}

\section{Complémentarité du $\mathrm{\rho} \rho$ et des traitements existants}

Face à l'émergence des bactéries antibiorésistantes, le $Q \rho$ apparaît comme une stratégie idéale pour élargir l'arsenal thérapeutique contre ces bactéries. Différents travaux ont montré que le QS était lié à la régulation de mécanismes impliqués dans la tolérance ou la résistance aux antimicrobiens. Par exemple, chez $P$. aeruginosa, le QS induit l'expression de peroxydases conférant aux bactéries une protection contre le peroxyde d'hydrogène et les antibiotiques de la famille des $\beta$-lactamines. Chez la même bactérie, il est également lié à la tolérance aux quinolones, à la tétracycline et à la kanamycine via l'induction de l'expression du régulateur transcriptionnel $n f x B$, un répresseur d'une pompe à efflux impliquée dans la résistance aux antibiotiques. En particulier, le biofilm, régulé par le QS [10], offre un environnement protecteur, favorisant une tolérance accrue aux agents antimicrobiens [32] $(\rightarrow)$. Ainsi l'utilisation du $\mathrm{Q} \rho$ permettrait d'augmenter la sensibilité aux

$(\rightarrow)$ Voir la synthèse de D. Lebeaux et J.M. Ghigo, $\mathrm{m} / \mathrm{s} \mathrm{n} \mathrm{n}^{\circ} 8-9$, août-septembre 2012, page 727 antimicrobiens. Par exemple, des effets synergiques entre $Q \rho$ et antibiothérapie ont été observés avec l'hydrate de baïcaline et la tobramycine chez $P$. aeruginosa 
et Burkholderia cepacia. Après exposition aux inhibiteurs du QS (PSI), les bactéries sont plus sensibles au traitement antibiotique in vitro mais également dans plusieurs modèles in vivo [33]. Outre les antibiotiques, une autre stratégie a été développée pour limiter la propagation des bactéries: la phagothérapie [34] $(\rightarrow)$.

Cette stratégie, délaissée dans un premier temps, $(\rightarrow)$ Voir la synthèse de N. Dufour et $L$. Debarbieux, $\mathrm{m} / \mathrm{s}^{\circ} 4$, avril 2017, page 410 suscite un regain d'intérêt depuis quelques années. Ce traitement consiste à utiliser les bactériophages, prédateurs naturels des bactéries, pour traiter les infections bactériennes. La phagothérapie est utilisée depuis près d'un siècle en Europe de l'Est, notamment en Géorgie et en Russie. Son utilisation a précédé celle des antibiotiques. Elle offre un traitement spécifique et personnalisable pour lutter contre les infections. Les bactéries ont néanmoins mis au point des mécanismes de défense et de résistance contre ces virus, dont certains sont sous le contrôle du QS. L'adsorption et la dégradation de plusieurs phages chez Vibrio cholerae semblent être soumises à la régulation du DS [35]. En effet, une souche de $V$. cholerae déficiente en QS présente une sensibilité accrue aux bactériophages par rapport à la souche sauvage [35]. Des études récentes ont mis en évidence des liens entre le QS et le système de défense bactérien CRISPR-Cas, qui permet aux bactéries de se prémunir contre les infections phagiques en intégrant des fragments d'ADN de phage dans leur génome pour renforcer leur « immunité » [36]. II a été montré que la baïcaline, un QSI, inhibe le système CRISPR-Cas chez $P$. aeruginosa [37].

L'inhibition de la communication bactérienne, entraînant une diminution de la virulence et de la formation de biofilm, mais également une augmentation de la sensibilité aux traitements antimicrobiens, présente donc des intérêts thérapeutiques très prometteurs en complément des thérapies existantes et pourrait permettre de contrer les phénomènes d'antibiorésistance.

\section{Les dispositifs médicaux}

Les dispositifs médicaux (ou DM) - comme les pansements, les cathéters, les endoscopes - sont des outils indispensables à la médecine moderne, mais ils peuvent constituer un point d'entrée pour les microorganismes pathogènes: on parle alors d'infections associées aux soins (IAS) ou infections nosocomiales [38]. Les DM constituent des supports sur lesquels les bactéries, notamment les espèces formant des biofilms, peuvent proliférer [32]. Une fois colonisé, le DM devient un réservoir infectieux pouvant héberger des bactéries parfois antibiorésistantes. Pour limiter ces IAS, de nouvelles stratégies visent à limiter la colonisation de ces dispositifs médicaux par les bactéries [32]. Parmi les solutions envisagées, le $Q \rho$ offre la possibilité de limiter la formation de biofilm, la tolérance aux antimicrobiens et la virulence des microbes $[16,26]$. Des QSI et des peptides inhibiteurs greffés à la surface des implants, de biomatériaux, de cathéters urinaires et sanguins, se sont montrés efficaces pour réduire la colonisation bactérienne in vivo et in vitro [16]. Les $\rho Q \varepsilon$ ont aussi fait l'objet de beaucoup d'attention pour la fonctionnalisation des dispositifs médicaux. Leur action extracellulaire leur permet d'agir sans contact direct avec les bactéries, en réduisant la concentration locale des Al. Des essais prometteurs ont été réalisés par incorporation d'enzymes dans les revêtements constituants les cathéters, les membranes, les capsules et les nanofibres, ainsi que pour d'autres applications thérapeutiques, comme des traitements topiques ou oraux par aérosolisation [16, 39].

\section{Autres applications du quorum quenching}

Si le blocage du QS présente des avantages évidents en santé humaine, d'autres domaines pourraient bénéficier des avancées de cette problématique (Figure 4). $\varepsilon n$ effet, les bactéries sont responsables d'infections, que ce soit chez les animaux ou chez les plantes, et les biofilms qu'elles forment posent des problèmes dans de nombreux secteurs [39]. Le rôle du QS dans les infections des poissons et des crevettes a, par exemple, été mis en évidence chez Vibrio spp. et Aeromonas spp. avec de sévères répercussions en aquaculture [40]. Chez les plantes, la bactérie Pectobacterium carotovorum (ou Erwinia carotovora) est responsable du feu bactérien, une maladie ciblant de nombreux arbres fruitiers engendrant chaque année de sérieuses conséquences en agriculture [39]. La formation des biofilms bactériens joue également un rôle prépondérant dans l'agrégation de salissures sur les surfaces immergées et les coques de bateaux, mieux connue sous le nom de biofouling [4l], dont les enjeux environnementaux sont considérables, et les conséquences économiques estimées à plusieurs dizaines de milliards d'euros chaque année [41].

\section{Conclusion}

Le QS module l'expression de nombreux gènes impliqués dans la sécrétion de toxines ou de facteurs de virulence, la compétence, la perméabilité membranaire, la motilité ou encore la formation de biofilm. Ce dernier contribuerait à de nombreux mécanismes impliqués dans la résistance aux agents antibactériens, en limitant leur diffusion ou en augmentant la fréquence des mutations des agents microbiens [42]. Compte tenu de son rôle prépondérant, à la fois dans la virulence et la résistance bactérienne, le QS est devenu une cible privilégiée pour le développement de nouvelles stratégies thérapeutiques pour répondre aux problèmes posés par I'utilisation massive d'antibiotiques. Des approches mettant en œuvre des inhibiteurs chimiques, des anticorps ou des enzymes ont été développées pour contrer les effets du QS [16]. Contrairement aux agents bactéricides, l'inhibition du QS cible le système de communication des bactéries et n'affecte pas directement leur survie. Ceci est particulièrement vrai pour les enzymes 


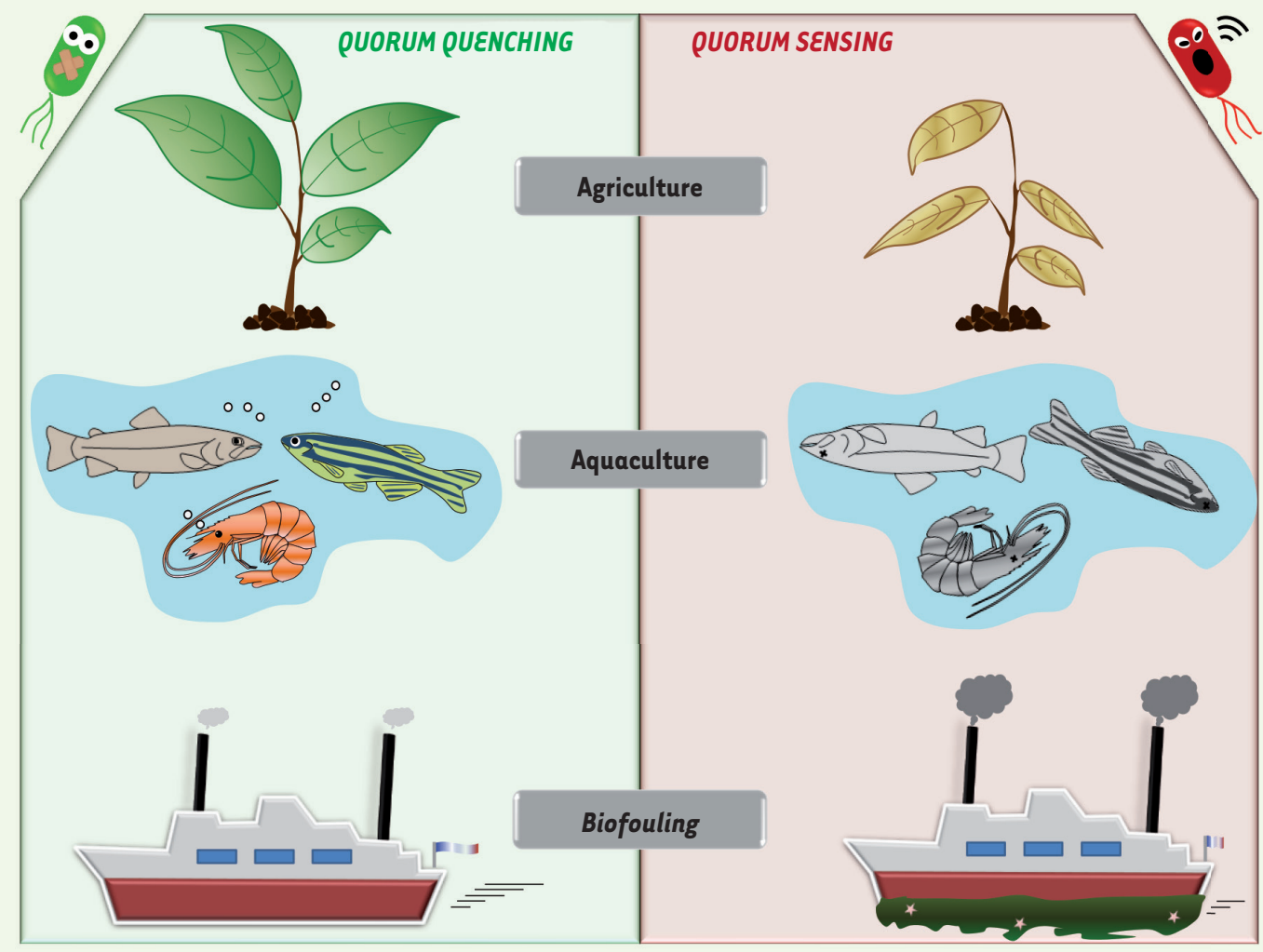

Figure 4. Le quorum quenching offre de nombreuses perspectives pour limiter les infections chez les plantes et les animaux ou pour lutter contre le biofouling. Outre ses nombreuses applications dans le domaine médical, le quorum quenching propose des solutions dans les domaines de l'aquaculture et l'agriculture pour prémunir les animaux et les plantes contre les infections bactériennes et ainsi limiter l'usage des antibiotiques dans ces domaines. Le quorum quenching pourrait aussi limiter le biofouling sur les coques de bateaux en agissant sur la première étape de l'apparition des salissures qui est le développement d'un biofilm bactérien.

qui agissent sur les molécules de communications sécrétées dans l'environnement. Certains inhibiteurs peuvent également montrer des effets biocides au-delà de certaines concentrations. L'effet synergique du $\rho \rho$ avec des traitements antibactériens tels que les antibiotiques, les antiseptiques ou les bactériophages ouvre donc la voie à des multithérapies.

Le $Q Q$ pourrait être à l'origine de nombreuses applications pour lutter contre les infections et pourrait permettre de réduire les doses d'antibiotiques utilisées en santé humaine et animale pour contrer les phénomènes de résistances bactériennes. Son efficacité contre la formation de biofilms constitue une alternative de choix aux agents biocides, tels que le cuivre utilisé notamment dans les peintures marines, qui s'accumule dans l'environnement et pose de sérieux problèmes de toxicité pour les écosystèmes marins. Le $Q Q$ se révèle être donc une nouvelle arme pour renforcer l'arsenal antibactérien disponible. L'efficacité du $Q \rho$ a été largement documentée, à la fois in vitro, in situ et in vivo, soulignant son énorme potentiel. Son développement nécessite désormais des phases pré-cliniques et cliniques plus approfondies pour répondre aux exigences réglementaires. Nul doute que le QQ constituera un axe de recherche thérapeutique majeur durant les prochaines années et que des investissements conséquents y seront consacrés tant les enjeux sanitaires, écologiques et économiques associés sont immenses. $\diamond$

\section{SUMMARY}

Quorum sensing and quorum quenching: how to disrupt bacterial communication to inhibit virulence? Most bacteria use a communication system known as quorum sensing which relies on the secretion and perception of small molecules called autoinducers enabling bacteria to adapt their behavior according to the population size and synchronize the expression of genes involved in virulence, antimicrobial resistance and biofilm formation. Methods have emerged to inhibit bacterial communication and limit their noxious traits. Chemical inhibitors, sequestering antibodies and degrading enzymes have been developed and proved efficient to decrease bacterial virulence both in vitro and in vivo. This strategy, named quorum quenching, also showed synergistic effects with traditional antibacterial treat- 
ments by increasing bacterial susceptibility to antibiotics. Thereby quorum quenching constitutes an interesting therapeutic strategy to fight against bacterial infections and limit the consequences of antibiotic resistance. $\diamond$

\section{REMERCIEMENTS}

Ce travail a été réalisé dans le cadre d'un contrat DGA-RAPID (LACTO-TEX) de la Direction générale de l'armement (DGA, France). Ce travail est également supporté par le programme «Investissements d'avenir» (Méditerranée Infection 10-IAHU-03) de l'Agence nationale de la recherche (ANR). B. Rémy est financé par une bourse de thèse du programme «Emplois Jeunes Doctorants» de la région Provence-Alpes-Côte d'Azur (PACA, France). S. Mion est financée par une bourse de thèse de la Direction générale de l'armement (DGA).

\section{LIENS D'INTÉRêT}

Laure Plener, Éric Chabrière, David Daudé ont des intérêts financiers dans l'entreprise Gene\&GreenTK.

Sonia Mion, Benjamin Rémy déclarent n'avoir aucun lien d'intérêt concernant les données publiées dans cet article.

\section{RÉFÉRENCES}

1. Bassler BL, Losick R. Bacterially speaking. Cell $2006 ; 125: 237-46$.

2. Federle MJ, Bassler BL. Interspecies communication in bacteria. J Clin Invest 2003 ; 112 : 1291-9.

3. Keller L, Surette MG. Communication in bacteria: an ecological and evolutionary perspective. Nat Rev Microbiol 2006 ; 4 : 249-58.

4. Tomasz A. Control of the competent state in Pneumococcus by a hormone-like cell product: An example for a new type of regulatory mechanism in bacteria. Nature $1965 ; 208: 155$.

5. Nealson KH, Platt T, Hastings I woodland. Cellular control of the synthesis and activity of the bacterial luminescent system. J Bacteriol $1970 ; 104: 313-22$.

6. Kaplan HB, Greenberg EP. Fischeri Luminescence System. J Bacteriol $1985 ; 163: 5$.

7. Nealson KH, Hastings JW. Bacterial bioluminescence: its control and ecological significance. Microbiol Rev 1979; $43:$ 496-518.

8. Fuqua WC, Winans $S C$, Greenberg EP. Quorum sensing in bacteria: the LuxR-Luxl family of cell density-responsive transcriptional regulators. J Bacteriol $1994 ; 176: 269-75$.

9. Miller MB, Bassler BL. Quorum sensing in bacteria. Annu Rev Microbiol 2001 ; 55 : 165-99.

10. Filloux A, Vallet I. Biofilm : mise en place et organisation d'une communauté bactérienne. Med/Sci (Paris) $2003 ; 19: 77-83$.

11. West SA, Winzer K, Gardner A, et al. Quorum sensing and the confusion about diffusion. Trends Microbiol 2012; $20: 586-94$.

12. Cornforth DM, Popat R, McNally L, et al. Combinatorial quorum sensing allows bacteria to resolve their social and physical environment. Proc Natl Acad Sci USA 2014 ; 111 : 4280-4.

13. Lee J, Zhang L. The hierarchy quorum sensing network in Pseudomonas aeruginosa. Protein Cell $2015 ; 6: 26-41$.

14. Papenfort K, Bassler BL. Quorum sensing signal-response systems in Gram-negative bacteria. Nat Rev Microbiol 2016 ; 14 : 576-88.

15. LaSarre B, Federle MJ. Exploiting quorum sensing to confuse bacterial pathogens. Microbiol Mol Biol Rev $2013 ; 77$ : 73-111.

16. Rémy B, Mion S, Plener L, et al. Interference in bacterial quorum sensing: A biopharmaceutical perspective. Front Pharmacol $2018 ; 9$.

17. Mashburn LM, Whiteley M. Membrane vesicles traffic signals and facilitate group activities in a prokaryote. Nature $2005 ; 437: 422-5$.

18. Pereira CS, Thompson JA, Xavier KB. Al-2-mediated signalling in bacteria. FEMS Microbiol Rev $2013 ; 37: 156-81$.

19. Tang K, Zhang X-H. Quorum quenching agents: Resources for antivirulence therapy. Mar Drugs $2014 ; 12: 3245-82$.

20. Park J, Jagasia R, Kaufmann GF, et al. Infection control by antibody disruption of bacterial quorum sensing signaling. Chem Biol 2007 ; 14 : 1119-27.
21. Kato N, Morohoshi T, Nozawa T, et al. Control of Gram-negative bacterial quorum sensing with cyclodextrin immobilized cellulose ether gel. J Incl Phenom Macrocycl Chem 2006 ; 56 : 55-9.

22. Fetzner S. Quorum quenching enzymes. J Biotechnol $2015 ; 201: 2-14$.

23. Defoirdt T, Boon N, Bossier P. Can bacteria evolve resistance to quorum sensing disruption? PLoS Pathog $2010 ; 6$ : e1000989.

24. Scutera S, Zucca M, Savoia D. Novel approaches for the design and discovery of quorum-sensing inhibitors. Expert Opin Drug Discov 2014 ; 9 : 353-66.

25. Ueda A, Attila C, Whiteley M, et al. Uracil influences quorum sensing and biofilm formation in Pseudomonas aeruginosa and fluorouracil is an antagonist. Microb Biotechnol $2009 ; 2: 62-74$.

26. Mion S, Rémy B, Plener L, et al. Empêcher les bactéries de communiquer : diviser pour mieux soigner. Ann Pharm Fr $2018 ; 76$ : 249-64.

27. Dong $\mathrm{YH}, \mathrm{Xu} J \mathrm{~L}$, Li XZ, et al. AiiA, an enzyme that inactivates the acylhomoserine lactone quorum-sensing signal and attenuates the virulence of Erwinia carotovora. Proc Natl Acad Sci USA 2000 ; 97 : 3526-31.

28. Weiland-Bräuer N, Kisch MJ, Pinnow N, et al. Highly effective inhibition of biofilm formation by the first metagenome-derived Al-2 quenching enzyme. Front Microbiol 2016 ; $7: 1098$.

29. Rémy B, Plener L, Poirier L, et al. Harnessing hyperthermostable lactonase from Sulfolobus solfataricus for biotechnological applications. Sci Rep $2016 ; 6: 37780$.

30. Hraiech S, Hiblot J, Lafleur J, et al. Inhaled Lactonase Reduces Pseudomonas aeruginosa Quorum Sensing and Mortality in Rat Pneumonia. PLoS One2014; 9 : el07125.

31. Guendouze A, Plener L, Bzdrenga J, et al. Effect of quorum quenching lactonase in clinical isolates of Pseudomonas aeruginosa and comparison with quorum sensing inhibitors. Front Microbiol $2017 ; 8: 227$.

32. Lebeaux D, Ghigo JM. Infections associées aux biofilms - Quelles perspectives thérapeutiques issues de la recherche fondamentale? Med Sc (Paris) $2012 ; 28: 727-39$.

33. Brackman G, Cos P, Maes L, et al. Quorum sensing inhibitors increase the susceptibility of bacterial biofilms to antibiotics in vitro and in vivo. Antimicrob Agents Chemother $2011 ; 55: 2655-61$.

34. Dufour N, Debarbieux L. La phagothérapie - Une arme crédible face à I'antibiorésistance. Med Sci (Paris) 2017 ; 33 : 410-6.

35. Hoque MM, Naser IB, Bari SMN, et al. Quorum regulated resistance of vibrio cholerae against environmental bacteriophages. Sci Rep 2016 ; 6 : 37956.

36. Patterson AG, Jackson SA, Taylor C, et al. Quorum sensing controls adaptive immunity through the regulation of multiple CRISPR-Cas systems. Mol Cell $2016 ; 64$ : 1102-8.

37. Høyland-Kroghsbo NM, Paczkowski J, Mukherjee S, et al. Quorum sensing controls the Pseudomonas aeruginosa CRISPR-Cas adaptive immune system. Proc Natl Acad Sci USA 2017 ; 114 : 131-5.

38. Neoh KG, Li M, Kang ET, et al. Surface modification strategies for combating catheter-related complications: recent advances and challenges. J Mater Chem B 2017 ; 5 : 2045-67.

39. Bzdrenga J, Daudé D, Rémy B, et al. Biotechnological applications of quorum quenching enzymes. Chem Biol Interact 2017 ; 267 : 104-15.

40. Defoirdt T, Sorgeloos P, Bossier P. Alternatives to antibiotics for the control of bacterial disease in aquaculture. Curr Opin Microbiol $2011 ; 14: 251-8$.

41. Lejars M, Margaillan A, Bressy C. Fouling release coatings: a nontoxic alternative to biocidal antifouling coatings. Chem Rev $2012 ; 112: 4347-90$.

42. Breidenstein EBM, Fuente-Núñez C de la, Hancock REW. Pseudomonas aeruginosa: all roads lead to resistance. Trends Microbiol $2011 ; 19$ : 419-26.

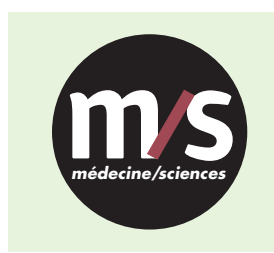

Tarifs d'abonnement m/s - 2019

$>$ Grâce à $m / s$, vivez en direct les progrès des sciences biologiques et médicales

Abonnez-vous

à médecine/sciences
Bulletin d'abonnement page 86 dans ce numéro de $\mathrm{m} / \mathrm{s}$
TIRÉS À PART

D. Daudé 